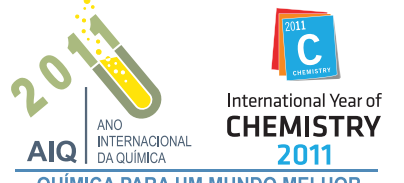

\title{
CONDIÇÕES DE EQUILÍBRIO TERMODINÂMICO: A FUNÇÃO DISPONIBILIDADE
}

\author{
Alessandro Ranulfo Lima Nery e Adalberto Bono Maurizio Sacchi Bassi* \\ Instituto de Química, Universidade Estadual de Campinas, CP 6154, 13084-862 Campinas - SP, Brasil
}

Recebido em 11/11/09; aceito em 25/6/10; publicado na web em 17/11/10

\begin{abstract}
CONDITIONS FOR THERMODYNAMIC EQUILIBRIUM: THE FUNCTION AVAILABILITY. The thermodynamic equilibrium is a state defined by conditions which depend upon some characteristics of the system. It requires thermal, mechanical, chemical and phase equilibrium. Continuum thermodynamics, its radical restriction usually called homogeneous processes thermodynamics, as well as the classical thermodynamic science of reversible processes, each of them defines equilibrium in a differing way. But these definitions lead to the same physical contents.
\end{abstract}

Keywords: thermodynamic equilibrium; continuum thermodynamics; availability function.

\section{INTRODUÇÃO}

Nos livros didáticos, a discussão sobre equilíbrio termodinâmico ocorre em duas partes, sendo a primeira feita a partir de três tipos particulares de equilíbrio, a saber, o térmico, o mecânico e o químico e de fases. Estes três tipos de equilíbrio são caracterizados por meio de propriedades termodinâmicas intensivas cujas homogeneidades (homogeneidade indica igualdade espacial, mas não necessariamente temporal) são exigidas, respectivamente, a temperatura, a pressão e os potenciais químicos de todas as espécies químicas presentes. O equilíbrio termodinâmico é apresentado como um estado em que os três equilíbrios acontecem simultaneamente, ou seja, um sistema será considerado em equilíbrio (neste trabalho, o termo equilíbrio será utilizado como sinônimo de equilíbrio termodinâmico) quando estiver em equilíbrio térmico, mecânico e químico e de fases.

Para a determinação do estado de equilíbrio, ou para a sua previsão, são usadas as condições de equilíbrio, as quais são apresentadas em termos de propriedades extensivas do sistema, sendo esta a segunda parte da discussão. Tais condições dependem das restrições às quais se encontrem submetidos o sistema e o processo que nele ocorra. Por outro lado, o estado de equilíbrio é, às vezes, classificado como estável, instável ou meta-estável. Esta classificação depende da resposta do sistema a pequenas perturbações que lhe sejam aplicadas. Tais perturbações, evidentemente, devem ser permitidas pelas mesmas restrições das quais dependem as condições de equilíbrio. Sempre que não adjetivado por estável, instável ou meta-estável, neste texto o substantivo equilíbrio admitirá qualquer uma destas três possibilidades.

Há a noção intuitiva de que, para que um sistema se encontre em equilíbrio, é necessário que os valores das suas propriedades permaneçam constantes no tempo. Neste contexto, deve-se ressaltar a diferença entre estado de equilíbrio e estado estacionário. Tanto no estado de equilíbrio quanto no estado estacionário, os valores das propriedades do sistema são temporalmente constantes. Mas, no equilíbrio, a homogeneidade das propriedades intensivas é necessária no sistema como um todo ou, pelo menos, em cada uma das fases (regiões homogêneas) que o constituem, enquanto que para o estado estacionário não há esta necessidade. Portanto, o estado de equilíbrio é um estado estacionário, mas o reverso nem sempre é verdade, porque o conceito de estado estacionário é mais abrangente.

*e-mail: bassi@iqm.unicamp.br
O estado estacionário que não for um estado de equilíbrio será denominado apenas estacionário. Logo, num estado apenas estacionário as propriedades intensivas serão temporalmente constantes, mas não serão homogêneas. Nem sempre, porém, é trivial a distinção entre estados de equilíbrio e estados apenas estacionários. De acordo com Levine, ${ }^{1}$ um sistema isolado estará em equilíbrio quando as suas propriedades estiverem constantes no tempo, enquanto que um sistema não isolado estará em equilíbrio quando as suas propriedades estiverem constantes no tempo e se, além disto, as suas propriedades também não se alterarem quando, por meio de isolamento e sem perturbação, o sistema for retirado do contato com as suas redondezas. Caso valha somente a primeira condição, o sistema não isolado estará em estado apenas estacionário.

Evidentemente, para um sistema isolado não existe a possibilidade de equilíbrio instável ou meta-estável, porque por definição tal sistema não permite trocas, de qualquer natureza, com o seu exterior, logo não pode ser perturbado, ou seja, uma vez em equilíbrio ele só pode permanecer neste estado. Além disto, a um sistema isolado não se aplica o conceito de estado apenas estacionário, porque não existe a possibilidade de verificar o seu comportamento num momento de não isolamento. Portanto, um sistema isolado ou está em equilíbrio estável, ou as suas propriedades estão se alterando com o passar do tempo.

Na termodinâmica clássica, o sistema é sempre formado por um número finito de fases. Por outro lado, na termodinâmica dos meios contínuos o valor de toda propriedade intensiva pode variar continuamente com a posição e com o tempo. Portanto, sendo $I$ uma propriedade intensiva, na termodinâmica dos meios contínuos pode-se escrever $I=I(\vec{x}, t)$, onde $\vec{x}$ é o vetor posição de um ponto pertencente ao sistema e $t$ é o tempo. Neste caso, nada impede que no sistema existam regiões finitas nas quais $I$ seja homogênea. Mas, de modo geral, isto é uma possibilidade, não uma exigência.

O objetivo deste trabalho é discutir o equilíbrio e as suas condições, sob o ponto de vista da termodinâmica dos meios contínuos e efetuar comparações com a termodinâmica clássica, por meio da termodinâmica dos processos homogêneos. Esta é a termodinâmica dos meios contínuos, sujeita à restrição de que ocorram somente processos homogêneos. Sem impor esta restrição, são também apresentadas noções básicas de termodinâmica dos meios contínuos, para exemplificar a utilidade da função disponibilidade. Este trabalho pretende contribuir ao ensino da termodinâmica. 


\section{O EQUILÍBRIO NA TERMODINÂMICA DOS MEIOS CONTÍNUOS}

Para que, no instante $t$, um sistema seja considerado em equilíbrio térmico, é necessário e suficiente que, neste instante, em todos os pontos geométricos do sistema a temperatura seja a mesma (nulidade da derivada parcial da temperatura em relação à posição, qualquer que seja a direção de alteração da posição, portanto homogeneidade térmica) e, além disto, a temperatura não tenda a variar no tempo (nulidade da derivada parcial em relação ao tempo, portanto constância temporal da temperatura). Note que as expressões equilíbrio térmico, homogeneidade térmica e constância temporal da temperatura no instante $t$ não indicam, respectivamente, o mesmo que equilíbrio térmico, homogeneidade térmica e constância temporal da temperatura ao longo de um intervalo temporal finito que contenha o instante $t$, por menor que seja tal intervalo finito. Note, também, que os conceitos de estável, instável ou meta-estável somente têm sentido se for possível conhecer o comportamento do sistema ao longo de um intervalo temporal finito, durante o qual, se desejado, seriam aplicáveis as pequenas perturbações que permitiriam efetuar esta classificação.

Como, sendo $T$ a temperatura do ponto $\vec{x}$ no instante $t$, tem-se $T=T(\vec{x}, t)$, a homogeneidade térmica e a constância temporal da temperatura do ponto $\vec{x}$, no instante $t$, podem ser, respectivamente, representadas por $\frac{\partial T(\vec{x}, t)}{\partial \vec{x}}=0$ e $\frac{\partial T(\vec{x}, t)}{\partial t}=0$. Se $\beta$ representar um sistema, lembrando que $\forall$ significa "para todo" e $\in$ indica "pertencente a", para que um sistema seja considerado em equilíbrio térmico no instante $t$ é, então, necessário e suficiente que

$$
\frac{\partial T(\vec{x}, t)}{\partial \vec{x}}=0=\frac{\partial T(\vec{x}, t)}{\partial t}, \forall \vec{x} \in \beta .
$$

Valendo, $\forall \vec{x} \in \beta$, a penúltima igualdade, o sistema será considerado termicamente homogêneo no instante $t \mathrm{e}$, valendo a última, termicamente estacionário no instante $t$. Portanto, para que ocorra equilíbrio térmico no instante $t$ é necessário e suficiente que o sistema seja termicamente homogêneo e termicamente estacionário naquele momento.

Para que ocorra equilíbrio térmico ao longo de um intervalo temporal finito $\Delta t$ (agora qualificável como estável, instável ou meta-estável, mas sendo a presente afirmação válida para as três qualificações) é necessário e suficiente que

$$
\frac{\partial T(\vec{x}, t)}{\partial \vec{x}}=0=\frac{\partial T(\vec{x}, t)}{\partial t}, \forall \vec{x} \in \beta \text { e } \forall t \in \Delta t .
$$

Conceitos análogos se aplicam aos equilíbrios mecânico e químico e de fases. Por exemplo, basta substituir a temperatura e suas derivadas, respectivamente, pela pressão $\mathrm{p}=\mathrm{p}(\vec{x}, \mathrm{t})$ e suas derivadas $\frac{\partial p(\vec{x}, t)}{\partial \vec{x}}$ e $\frac{\partial p(\vec{x}, t)}{\partial t}$, para se chegar à condição necessária e suficiente à ocorrência de equilíbrio mecânico no instante $t$. Obtém-se, assim,

$$
\frac{\partial p(\vec{x}, t)}{\partial \vec{x}}=0=\frac{\partial p(\vec{x}, t)}{\partial t}, \forall \vec{x} \in \beta,
$$

correspondendo a penúltima igualdade ao sistema baricamente homogêneo no instante $t$ e a última, ao baricamente estacionário naquele momento.

Semelhantemente, para haver equilíbrio químico e de fases no instante $t$, a temperatura e suas derivadas devem ser respectivamente substituídas pelos potenciais químicos $\mu_{j}=\mu_{j}(\vec{x}, t), j=1, \ldots, J$ e suas derivadas $\frac{\partial \mu_{j}(\vec{x}, t)}{\partial \vec{x}}, j=1, \ldots, J$ e $\frac{\partial \mu_{j}(\vec{x}, t)}{\partial t}, j=1, \ldots, J$, onde $J$ é o número de espécies químicas presentes no sistema e $j=1, \ldots$,
$J$ indica qual, entre as $J$ espécies químicas, está sendo considerada. Obtém-se, assim, a condição necessária e suficiente

$$
\frac{\partial \mu_{j}(\vec{x}, t)}{\partial \vec{x}}=0=\frac{\partial \mu_{j}(\vec{x}, t)}{\partial t}, \forall j \in(1, \ldots, J) \text { e } \forall \vec{x} \in \beta,
$$

onde $(1, \ldots, J)$ representa o conjunto ordenado de valores $j$. Valendo, $\forall \vec{x} \in \beta$, o conjunto das $J$ penúltimas igualdades, uma para cada um dos $J$ possíveis valores de $j$, o sistema será considerado quimicamente homogêneo no instante $t$ e, valendo o conjunto das $J$ últimas, quimicamente estacionário naquele momento.

Para que um sistema esteja em equilíbrio no instante $t$, é necessário e suficiente que o sistema seja térmica, mecânica e quimicamente homogêneo e estacionário naquele momento. Analogamente, para que um sistema esteja em equilíbrio ao longo de um intervalo temporal finito $\Delta t$ é necessário e suficiente que

$$
\begin{aligned}
& \frac{\partial T(\vec{x}, t)}{\partial \vec{x}}=0=\frac{\partial T(\vec{x}, t)}{\partial t}, \frac{\partial p(\vec{x}, t)}{\partial \vec{x}}=0=\frac{\partial p(\vec{x}, t)}{\partial t} \mathrm{e} \\
& \frac{\partial \mu_{j}(\vec{x}, t)}{\partial \vec{x}}=0=\frac{\partial \mu_{j}(\vec{x}, t)}{\partial t}, \forall j \in(1, \ldots, J), \forall \vec{x} \in \beta \text { e } \forall t \in \Delta t .
\end{aligned}
$$

Mas a satisfação destas $2 J+4$ condições é suficiente para garantir o equilíbrio estável somente no caso de sistema isolado. Isto é o mesmo que afirmar que, para garantir a estabilidade do equilíbrio, adicionalmente a estas $2 J+4$ condições deve ser imposta a impossibilidade de trocas mássicas com o exterior em qualquer momento (sistema fechado) e, sendo $\frac{d Q(t)}{d t}$ e $\frac{d W(t)}{d t}$, respectivamente, a potência térmica e atérmica trocada pelo sistema com o seu exterior no instante $t$, também deve ser imposto que

$$
\frac{d Q(t)}{d t}=0=\frac{d W(t)}{d t}, \forall t \in \Delta t,
$$

porque estas três imposições adicionais isolam o sistema durante o intervalo $\Delta t$.

Cabe, ainda, ressaltar que todo sistema térmica, bárica e quimicamente homogêneo no instante $t$ é, neste momento, homogêneo (para todas as suas propriedades intensivas) em relação ao sistema como um todo ou, pelo menos, em cada uma das fases que o constituem. Analogamente, todo sistema fechado térmica, bárica e quimicamente estacionário no instante $t$ é, neste momento, estacionário (para todas as suas propriedades). Portanto, se $E=E(t)$ for uma propriedade extensiva qualquer, em todo sistema fechado térmica, bárica e quimicamente estacionário no instante $t$ ter-se-á $\frac{d E(t)}{d t}=0$ neste momento, logo, a satisfação desta igualdade será necessária para que, no instante considerado, o sistema esteja em equilíbrio. Mas as condições suficientes são as condições de equilíbrio mencionadas no início do segundo parágrafo da Introdução, as quais são fornecidas em termos de propriedades extensivas específicas. Portanto, em geral um arbitrário conjunto de condições $\frac{d E(t)}{d t}=0$ não é suficiente para

\section{COMPARAÇÃO COM A TERMODINÂMICA CLÁSSICA}

Antes de avançar mais, convém traçar um paralelo entre a termodinâmica dos meios contínuos e a termodinâmica clássica. Para isto, considere que um ponto bem determinado corresponda ao instante $t \in \Delta t$, num gráfico em que o valor da pressão de um sistema baricamente homogêneo no momento $t$ seja lançado como ordenada, enquanto que o valor do volume do sistema, no mesmo instante, seja marcado como abscissa. Como, para que ocorra equilíbrio mecânico 
ao longo do intervalo $\Delta t$, é necessário e suficiente que

$$
\frac{\partial p(\vec{x}, t)}{\partial \vec{x}}=0=\frac{\partial p(\vec{x}, t)}{\partial t}, \forall \vec{x} \in \beta \text { e } \forall t \in \Delta t,
$$

um segmento de curva que contenha tal ponto não corresponde à conservação, ao longo de $\Delta t$, de qualquer tipo de equilíbrio mecânico (estável, instável ou meta-estável), a não ser que se trate de um segmento de reta horizontal (como acontece numa transição de fase).

Por isto, tal segmento de curva, em geral, representa uma sequência de estados baricamente homogêneos cuja pressão varia continuamente, chamada processo baricamente homogêneo. Um processo baricamente homogêneo pode ser entendido como o limite ao qual tenderá um processo real, quando tender para zero a razão entre a velocidade de avanço do processo e a velocidade de homogeneização da pressão no sistema. Embora, a rigor, nenhum processo baricamente homogêneo seja fisicamente realizável, muitos processos são baricamente homogêneos dentro do limite de precisão experimental desejado (pense, por exemplo, na efusão de um gás). Processos térmica ou quimicamente homogêneos são definidos de modo semelhante.

Chama-se processo homogêneo a um processo térmica, bárica e quimicamente homogêneo. Portanto, ao longo de um processo homogêneo o sistema permanece homogêneo (em relação a todas as suas propriedades intensivas), no sistema como um todo ou, pelo menos, em cada uma das fases que o constituem. Os processos homogêneos não dissipativos apresentam o mesmo formalismo matemático dos processos reversíveis da termodinâmica clássica. Por isto, os processos homogêneos não dissipativos fornecem a ligação entre a termodinâmica dos meios contínuos e a termodinâmica clássica.

Como a termodinâmica clássica não utiliza o tempo como uma de suas variáveis, as derivadas temporais antes apresentadas são substituídas por informações relativas ao exterior do sistema. O equilíbrio mecânico será usado para exemplificar a substituição feita pela termodinâmica clássica, ocorrendo uma substituição análoga em cada um dos outros dois equilíbrios. Para efetuar esta específica exemplificação, deve-se inicialmente definir a pressão de oposição (ou do exterior) $p^{\prime}$, homogeneamente aplicada, pelo exterior do sistema, sobre a superfície fechada que delimita o mencionado sistema. Se, num determinado momento, a pressão do sistema for homogênea e igual a $p^{\prime}$, naquele instante o sistema, além de baricamente homogêneo, também será baricamente estacionário (logo, estará em equilíbrio mecânico). Reciprocamente, se em determinado momento o sistema for baricamente homogêneo e estacionário (logo, estiver equilíbrio mecânico), naquele instante a pressão do sistema será igual a $p^{\prime}$.

\section{CONDIÇÕES DE EQUILÍBRIO DA TERMODINÂMICA DOS PROCESSOS HOMOGÊNEOS}

A determinação do estado de equilíbrio, efetuada por meio das condições de equilíbrio, depende do tipo de processo e das condições nas quais esse processo ocorra, condições estas definidas pelo tipo de sistema. Além disso, as condições de equilíbrio determinam o caminho a ser seguido pelo sistema, até ser atingido o equilíbrio. De acordo com Atkins, ${ }^{2}$ mas utilizando a mencionada analogia de formalismo matemático entre os processos reversíveis da termodinâmica clássica e os processos homogêneos não dissipativos, ${ }^{3}$ para um sistema fechado cuja energia atérmica trocada com seu exterior seja somente trabalho volumétrico expansivo, (a) dados a energia interna $\mathrm{U}$ e o volume $\mathrm{V}$, no equilíbrio a entropia $\mathrm{S}$ é máxima; (b) dados $\mathrm{S}$ e $\mathrm{V}$, no equilíbrio U é mínima; (c) dados $\mathrm{S}$ e a pressão homogênea $\mathrm{P}$, no equilíbrio a entalpia $\mathrm{H}$ é mínima; (d) dados $\mathrm{H}$ e $\mathrm{P}$, no equilíbrio $\mathrm{S}$ é máxima; (e) dados $\mathrm{V}$ e a temperatura homogênea $\mathrm{T}$, no equilíbrio a energia de Helmholtz A é mínima; (f) dados T e P, no equilíbrio a energia de Gibbs G é mínima.
No parágrafo anterior, por meio da analogia entre a termodinâmica clássica e a restrição da termodinâmica dos meios contínuos denominada termodinâmica dos processos homogêneos, as condições de equilíbrio relativas a esta última teoria foram fornecidas. Evidentemente, cabe agora demonstrar como a termodinâmica dos meios contínuos, ao sofrer a mencionada restrição, atinge as conclusões citadas no parágrafo anterior. Especificamente para as situações (a) e (e), isto é mostrado na última seção anterior às Conclusões. Para não alongar demasiadamente o texto, para as demais demonstrações prefere-se fazer referência aos livros de Truesdell ${ }^{3}$ e Liu. ${ }^{4}$

Note que, conforme antecipado, em todos os casos mencionados é sempre uma propriedade extensiva, $E=E(t)$, que indica se o sistema se encontra, ou não, em equilíbrio. Por exemplo, o caso (a) se refere a um sistema isolado, onde a entropia é máxima no equilíbrio, o que pode ser expresso por meio de $\frac{d S(t)}{d t}=0$. Mas, enquanto que a derivada temporal da entropia somente é nula no equilíbrio, as derivadas temporais de outras propriedades extensivas do sistema isolado como, por exemplo, o volume e a energia interna são nulas mesmo fora do equilíbrio, confirmando que, em geral, $\frac{d E(t)}{d t}=0$ não é suficiente para garantir que o sistema se encontre em equilíbrio. Para os demais casos, comentários análogos podem ser feitos.

Note, também, que não foi especificado se o equilíbrio é estável, instável ou meta-estável. Para efetuar esta classificação, imagine uma superfície que forneça o valor da variável extensiva a ser extremada no equilíbrio, em função das propriedades que definem o estado do sistema. De acordo com a termodinâmica dos processos homogêneos, quando se tratar de um extremo absoluto desta superfície, o equilíbrio será estável. Quando se tratar de um extremo relativo, superável desde que uma barreira seja ultrapassada, o equilíbrio será meta-estável. Quando se tratar de um ponto de sela, o equilíbrio será instável.

\section{A FUNÇÃO DISPONIBILIDADE}

As bem conhecidas condições de equilíbrio apresentadas na seção anterior limitam-se à termodinâmica dos processos homogêneos, a qual é uma radical simplificação da termodinâmica dos meios contínuos, simplificação esta que corresponde a uma situação extremamente específica. Para abranger a termodinâmica dos meios contínuos, pode-se considerar a existência, em qualquer sistema, de uma propriedade extensiva originalmente proposta por Liapounov. ${ }^{5}$ Tal propriedade, chamada disponibilidade (availability), será neste texto representada por $F$. Quando o sistema espontaneamente (sem que energia lhe seja fornecida) alterar o seu estado em direção ao estado de equilíbrio, por definição a velocidade de consumo de $F$ pelo sistema, a cada instante, será uma medida da velocidade de avanço do sistema naquele momento.

Como acontece com toda propriedade extensiva do sistema, evidentemente $F=F(t)$. Sempre que o sistema alterar o seu estado em direção ao estado de equilíbrio, por definição ter-se-á $\frac{d F(t)}{d t} \leq 0$, sendo a igualdade válida somente para o estado de equilíbrio. Logo, por definição $F$ descreve as condições de equilíbrio para o tipo de processo e de sistema considerados. Por exemplo, no caso de sistema isolado a disponibilidade é o simétrico da entropia, ou seja, $F=-S$. Mas, se o sistema não for isolado, $F \neq-S$.

\section{NOÇÕES DE TERMODINÂMICA DOS MEIOS CONTÍNUOS}

Um meio será considerado contínuo quando a densidade volumétrica da sua massa variar espacialmente de modo contínuo ou, 
pelo menos, for espacialmente integrável. ${ }^{4} \mathrm{Na}$ termodinâmica dos meios contínuos utilizam-se as densidades mássicas das propriedades extensivas, ou seja, sempre se trabalha com propriedades intensivas e, para se obter as propriedades extensivas referentes ao sistema todo ou a uma parte finita dele, efetua-se a integral, sobre o volume do corpo, do produto da densidade mássica da propriedade considerada pela densidade volumétrica da massa. Por exemplo, seja $Y=Y(t)$ o valor de uma propriedade extensiva no instante $t$, para o sistema como um todo e seja $y=y(\vec{x}, t)$ a sua densidade mássica no ponto $\vec{x}$, neste mesmo momento. O valor $Y=Y(t)$ será dado por $Y(t)=\int_{V(t)} \rho(\vec{x}, t) y(\vec{x}, t) d V$, onde $\rho=\rho(\vec{x}, t)$ é a densidade volumétrica de massa no ponto $\vec{x}$, no instante $t$ e $V(t)$ é o volume do sistema neste momento.

A equação de balanço ${ }^{4}$ de $Y(t)=\int_{V(t)} y_{V}(\vec{x}, t) d V$, sendo $y_{V}(\vec{x}, t)=\rho(\vec{x}, t) y(\vec{x}, t)$ é

$$
\frac{d}{d t} \int_{V(t)} y_{V} d V=\int_{\partial V(t)} \Phi_{y} \vec{n} d a+\int_{V(t)} \sigma_{y} d V
$$

onde $\frac{d}{d t} \int_{V(t)} y_{V} d V$ é a velocidade de variação do valor $Y$, referente ao volume $V(t)$, no instante $t, \int_{\partial V(t)} \Phi_{y} \vec{n} d a$ é a velocidade de transferência de $Y$, no instante $t$, através da superfície fechada $\partial V(t)$ que envolve o volume $V(t)$ e $\int_{V(t)} \sigma_{y} d V$ é a velocidade de criação ou aniquilação de $Y$ no instante $t$, dentro do volume $V(t)$. A grandeza $-\Phi_{y}$ (o sinal negativo provém da convenção de que é positivo o fluxo que entra no volume $V$, enquanto que $\vec{n}$ é um vetor unitário perpendicular à superfície e dirigido para fora de $V$ ) é chamada fluxo de $Y$, enquanto que $\sigma_{y}$ é o suprimento de $Y$. Se $Y$ for um escalar, $y_{V}$ e $\sigma_{y}$ também serão escalares, enquanto que $\Phi_{y}$ será um vetor. Se $Y$ for um vetor, $y_{V}$ e $\sigma_{y}$ também serão vetores, enquanto que $\Phi_{y}$ será um tensor de segunda ordem (matriz quadrada). $\mathrm{O}$ suprimento de $Y$ tanto pode ser devido a fontes externas ao volume $V(t)$, quanto pode ser originado por movimentos internos ao volume $V(t)$.

São chamadas conservativas as grandezas para as quais os correspondentes suprimentos são devidos exclusivamente a fontes externas. A energia total é um exemplo de grandeza conservativa, porque seu suprimento é o produto da densidade volumétrica de massa, $\rho$, pela soma $\vec{x} \cdot \vec{b}+\mathrm{r}$, ou seja, $\sigma_{\mathrm{e}}=(\rho)(\vec{x} \cdot \vec{b}+\mathrm{r})$, onde o escalar $r(\vec{x}, t)$ é uma densidade mássica de potência térmica causada por fonte externa (por exemplo, causada por uma radiação absorvida) e o vetor $b(\vec{x}, t)$ é uma densidade mássica de força, também causada por fonte externa (por exemplo, causada pelo campo gravitacional da Terra). Por outro lado, o fluxo de energia total, $-\Phi_{e}$, é dado por $\Phi_{e}=T \dot{\vec{x}}-\vec{q}$, onde $T(\vec{x}, t)$ é o tensor de tração de Cauchy, $\dot{\vec{x}}(\vec{x}, t)$ é a velocidade, $T \dot{\vec{x}}$ é o simétrico do fluxo de potência mecânica e $q(\vec{x}, t)$ é o fluxo de potência térmica. A energia total costuma ser decomposta na soma da energia cinética, $\int_{V(t)} \frac{\rho}{2} \dot{\vec{x}} \cdot \dot{\vec{x}} d V$, com a energia interna, $\int_{V(t)} \rho u d V$, onde $u$ é a densidade mássica de energia interna. Portanto, o balanço de energia total é fornecido pela igualdade

$$
\frac{d}{d t} \int_{V(t)} \rho\left(\frac{\dot{\vec{x}} \cdot \dot{\vec{x}}}{2}+u\right) d V=\int_{\partial V(t)}(T \dot{\vec{x}}-\vec{q}) \cdot \vec{n} d a+\int_{\mathrm{V}(t)} \rho(\dot{\vec{x}} \cdot \vec{b}+r) d V .
$$

O suprimento de energia cinética provém de $\vec{b}$, mas, também, de uma densidade volumétrica de potência deformativa cujo efeito é diminuir este suprimento. O suprimento de energia interna provém de $r$, mas, também, da mencionada densidade volumétrica de potência deformativa, cujo efeito é aumentar este suprimento. Como a diminuição no suprimento de energia cinética e o aumento no suprimento de energia interna têm igual módulo, a densidade volumétrica de potência deformativa não afeta o suprimento de energia total. A potência deformativa é produzida internamente no sistema. A potência deformativa, portanto, não é um suprimento proveniente de fonte externa, o que indica que a energia cinética e a energia interna não são conservativas. ${ }^{4}$

Demonstra-se que ${ }^{4}$

$$
\begin{aligned}
\frac{d}{d t} \int_{V(t)} \frac{\rho}{2}(\dot{\vec{x}} \cdot \dot{\vec{x}}) d V & =\int_{\partial V(t)} T \dot{\vec{x}} \cdot \vec{n} d a+\int_{V(x)}(\rho \dot{\vec{x}} \cdot \vec{b}-T \cdot \operatorname{grad} \dot{\vec{x}}) d V(10) \\
\mathrm{e} \frac{d}{d t} \int_{V(t)} \rho u d V & =-\int_{\partial V(t)} \vec{q} \cdot \vec{n} d a+\int_{V(t)}(\rho r+T \cdot \operatorname{grad} \dot{\vec{x}}) d V
\end{aligned}
$$

são, respectivamente, a equação de balanço da energia cinética e da energia interna. Nestas equações, grad $\dot{\vec{x}}$ é o tensor gradiente do vetor velocidade e a contração tensorial $T \cdot \operatorname{grad} \dot{\vec{x}}$ produz um escalar, o qual é o valor da potência deformativa em cada instante $t$ e em cada ponto $\vec{x}$ do sistema.

Outro exemplo de grandeza não conservativa é a entropia, dada pela equação $S=\int_{V(t)} \rho s d V$, sendo $s$ a densidade mássica de entropia. A velocidade de variação da entropia é a soma da velocidade de troca de entropia com o exterior, $\dot{S}_{\text {troc}}$, com a velocidade de produção de entropia no interior do corpo, $\dot{S}_{\text {prod }}$, ou seja, $\frac{d S}{d t}=\dot{S}_{\text {troc }}+\dot{S}_{\text {prod }}$. A velocidade de troca de entropia com o exterior é dada por $\dot{S}_{\text {troc }}=-\int_{\partial V(t)} \vec{\Phi}_{S} \cdot \vec{n} d a+\int_{V(t)} \rho \sigma_{S} d V$, sendo $\vec{\Phi}_{\mathrm{s}}$ o fluxo de entropia e $\sigma_{\mathrm{s}}$ o suprimento mássico de entropia devido a causas externas ao sistema, o que mostra que $S_{\text {troc }}$ é conservativa. Portanto, o balanço de entropia pode ser escrito

$$
\dot{S}_{p r o d}=\frac{d S}{d t}-\dot{S}_{t r o c}=\frac{d}{d t} \int_{V(t)} \rho s d V+\int_{\partial V(t)} \vec{\Phi}_{S} \cdot \vec{n} \mathrm{da}-\int_{V(t)} \rho \sigma_{S} d V .
$$

Vale ressaltar que a entropia $S$ não é conservativa, porque $\dot{S}_{\text {prod }}$ é uma velocidade de criação de entropia causada por um suprimento produzido internamente no sistema.

Impondo, ao balanço de entropia, a segunda lei da termodinâmica $\dot{S}_{\text {prod }} \geq 0$, tem-se

$$
\frac{d}{d t} \int_{V(t)} \rho s d V+\int_{\partial V(t)} \vec{\Phi}_{S} \cdot \vec{n} \mathrm{da}-\int_{V(t)} \rho \sigma_{S} d V \geq 0,
$$

expressão esta conhecida como a desigualdade da entropia. Para várias aplicações é válido considerar que, em cada ponto do sistema, o fluxo de entropia, $\vec{\Phi}_{\mathrm{s}}$ e o suprimento mássico de entropia, $\sigma_{\mathrm{s}}$, são respectivamente proporcionais ao fluxo térmico $\vec{q}$ e à densidade mássica de potência térmica $r$, sendo a constante de proporcionalidade dada pelo recíproco da temperatura absoluta no ponto considerado, ou seja, ${ }^{3,4} \quad \vec{\Phi}_{s}=\frac{1}{T} \vec{q}$ e $\sigma_{s}=\frac{1}{T} r$. Estas duas últimas equações, porém, são reconhecidamente inconsistentes com resultados da teoria cinética dos gases, ${ }^{4,6}$ portanto não apresentam validade geral. Mas, sempre que elas puderem ser consideradas corretas (por exemplo, para aplicações em engenharia dos materiais, onde inclusive fundamentam vários softwares computacionais), tornar-se-á conveniente a substituição das mesmas na desigualdade da entropia, o que produzirá a desigualdade de Clausius-Duhem ${ }^{7}$

$$
\frac{d}{d t} \int_{V(t)} \rho s d V+\int_{\partial V(t)} \frac{1}{T} \vec{q} \cdot \vec{n} d a-\int_{V(t)} \frac{1}{T} \rho r d V \geq 0 .
$$

\section{Exemplos de disponibilidade na termodinâmica dos meios contínuos}

Suponha a inexistência de fontes externas de suprimento $(\vec{b}=0=r)$ e, além disto, que a superfície do sistema seja fixa ( $\dot{\vec{x}}=0$ na superfície) e adiabática ( $\vec{q} \cdot \vec{n}=0$ na superfície). Neste caso, a Equação 9 , 
para balanço de energia total, se reduz a $\frac{d}{d t} \int_{V(t)} \rho\left(\frac{\dot{\vec{x}} \cdot \dot{\vec{x}}}{2}+u\right) d V=0$, o que significa que a energia total do sistema é constante, enquanto que a Equação 14, que é a desigualdade de Clausius-Duhem, se reduz a $\frac{d}{d t} \int_{V(t)} \rho s d V \geq 0$, o que significa que a entropia não diminui. Note que um sistema isolado é tal que sua superfície é fixa, adiabática e isolante em relação a qualquer fonte externa de suprimento. Portanto, neste caso a disponibilidade $F(t)=-\int_{V(t)} \rho s d V$, porque $\frac{d F(t)}{d t} \leq 0$, em concordância com a termodinâmica dos processos homogêneos (e com a termodinâmica clássica).

Como segundo exemplo, novamente suponha a inexistência de fontes externas de suprimento $(\vec{b}=0=r)$, mas considere uma superfície fixa ( $\dot{\vec{x}}=0$ na superfície) e isotérmica (temperatura homogênea e constante no tempo, o que é representado por $T=T_{\mathrm{o}}$ na superfície). Neste caso, $\frac{d}{d t} \int_{V(t)} \rho\left(\frac{\dot{\vec{x}} \cdot \overrightarrow{\vec{x}}}{2}+u\right) d V+\int_{\partial V(t)} \vec{q} \cdot \vec{n} d a=0$ será o balanço de energia total, enquanto que $\frac{d}{d t} \int_{V(t)} \rho s d V+\frac{1}{T_{\mathrm{o}}} \int_{\partial V(t)} \vec{q} \cdot \vec{n} d a \geq 0$ será a desigualdade de Clausius-Duhem, o que produz

$\frac{d}{d t} \int_{V(t)} \rho T_{\mathrm{o}} s d V-\frac{d}{d t} \int_{V(t)} \rho\left(\frac{\dot{\vec{x}} \cdot \dot{\vec{x}}}{2}+u\right) d V \geq 0$.

Portanto, se $F(t)=\int_{V(t)} \rho\left(u-T_{\mathrm{o}} s+\frac{\dot{\vec{x}} \cdot \dot{\vec{x}}}{2}\right) d V$, tem-se $\frac{d F(t)}{d t} \leq 0$.

Como caso especial, suponha que não apenas a superfície do sistema, mas sim que ele todo seja isotérmico. Logo, suponha $T=T_{\mathrm{o}}$ no sistema. Neste caso, $A(t)=\int_{V(t)} \rho\left(u-T_{\mathrm{o}} \mathrm{s}\right) d V$ será a energia de Helmholtz do sistema, portanto $F(t)=A(t)+\int_{V(t)} \frac{\rho}{2}(\dot{\vec{x}} \cdot \dot{\vec{x}}) d V$. Mas, para um sistema fixo isotérmico, sua energia cinética $\int_{V(t)} \frac{\rho}{2}(\dot{\vec{x}} \cdot \dot{\vec{x}}) d V$ é uma constante, portanto $F(t)$ e $A(t)$ diferem apenas por uma constante aditiva. Logo, neste caso especial $A(t)$ deve ser mínimo no equilíbrio, novamente em concordância com a termodinâmica dos processos homogêneos (e com a termodinâmica clássica).

\section{CONCLUSÃO}

Neste trabalho, dentro do contexto da discussão do equilíbrio termodinâmico e da maior divulgação da relativamente pouco conhecida função disponibilidade, também foram apresentadas noções básicas de termodinâmica dos meios contínuos. Elas mostram como as duas principais leis da termodinâmica se inserem no contexto da mecânica. De fato, o balanço de energia total, dado pela Equação 9, bem como os balanços de energia cinética e interna dados respectivamente pelas Equações 10 e 11, são absolutamente coerentes com os balanços de momento linear e angular (não apresentados neste trabalho). ${ }^{4} \mathrm{O}$ balanço de entropia, dado pela Equação 12, é absolutamente coerente com a equação geral de balanço das grandezas mecânicas, dado pela Equação 8. Para se obter a desigualdade da entropia, dada pela Equação 13 , basta impor $\dot{S}_{\text {prod }} \geq 0$ à Equação 12. Esta imposição, junto com as igualdades $\vec{\Phi}_{S}=\frac{1}{T} \vec{q}$ e $\sigma_{S}=\frac{1}{T} r$ (estas últimas com limites de validade ainda a serem claramente estabelecidos), são os únicos verdadeiros acréscimos à mecânica.

A inserção da termodinâmica dos meios contínuos na mecânica é tão forte que, hoje em dia, a mecânica dos meios contínuos costuma incluir esta teoria termodinâmica. Por exemplo, os títulos dos livros de Liu, ${ }^{4}$ Dill, ${ }^{8}$ Chung, ${ }^{9}$ Irgens ${ }^{10}$ e Nair ${ }^{11}$ não envolvem a palavra termodinâmica, embora incluam toda a termodinâmica dos meios contínuos. A termodinâmica dos processos homogêneos é uma radical restrição à termodinâmica dos meios contínuos que apresenta, em relação a esta última, a desvantagem da imposição de homogeneidade às propriedades intensivas, o que a afasta da realidade dos processos naturais. Mas ela apresenta, como vantagens em relação à termodinâmica dos meios contínuos, os fatos de ser matematicamente muito menos complexa e formalmente semelhante à termodinâmica clássica. Ela é a teoria temporal que interliga as outras duas teorias termodinâmicas.

A termodinâmica clássica é a única, entre todas as ciências naturais, que omite a grandeza tempo, além de também ser a única, entre as três teorias termodinâmicas mencionadas, que apenas é aplicável a processos cujos respectivos estados iniciais e finais sejam estados de equilíbrio. Entretanto, este trabalho mostra que, como seria de se esperar, os enfoques do equilíbrio termodinâmico são coerentes, quer se trate da termodinâmica clássica, da termodinâmica dos processos homogêneos ou da termodinâmica dos meios contínuos. Entender as teorias mais abrangentes permite uma mais clara compreensão das mais restritivas. Por outro lado, conhecer as teorias mais restritivas é utilíssimo para a interpretação física dos resultados matemáticos das mais abrangentes. Estas duas afirmações são especialmente verdadeiras quando se procura aprimorar conceitualmente a idéia intuitiva de equilíbrio.

\section{REFERÊNCIAS}

1. Levine, I. N.; Physical Chemistry, Tata McGraw - Hill: New Delhi, 2002.

2. Atkins, P. W.; Físico Química, L.T.C.: Rio de Janeiro, 1999.

3. Truesdell, C. A.; Rational Thermodynamics, Springer: Berlin, 1984

4. Liu, I-S.; Continuum Mechanics, Springer: Berlin, 2002.

5. Knops, R. J.; Wilkes, E. W.; Handbuch der Physik, Springer: Berlin, 1973.

6. Muller, I.; Ruggeri, T.; Rational Extended Thermodynamics, Springer: Berlin, 1998.

7. Truesdell, C. A.; Toupin, R.; Handbuch der Physik, Springer: Berlin, 1960.

8. Dill, E. H.; Continuum Mechanics: Elasticity, Plasticity, Viscoelasticity, CRC: London, 2006.

9. Chung, T. J.; General Continuum Mechanics, Cambridge: Cambridge, 2007.

10. Irgens, F.; Continuum Mechanics, Springer: Berlin, 2008.

11. Nair, S.; Introduction to Continuum Mechanics, Cambridge: Cambridge, 2009. 